\title{
Targeted gene disruption by use of transcription activator-like effector nuclease (TALEN) in the water flea Daphnia pulex
}

\author{
Chizue Hiruta' ${ }^{1}$, Yukiko Ogino ${ }^{1,2}$, Tetsushi Sakuma ${ }^{3}$, Kenji Toyota ${ }^{1,2}$, Shinichi Miyagawa ${ }^{1,2}$, Takashi Yamamoto ${ }^{3}$ \\ and Taisen Iguchi , $^{*}$
}

\begin{abstract}
Background: The cosmopolitan microcrustacean Daphnia pulex provides a model system for both human health research and monitoring ecosystem integrity. It is the first crustacean to have its complete genome sequenced, an unprecedented ca. $36 \%$ of which has no known homologs with any other species. Moreover, D. pulex is ideally suited for experimental manipulation because of its short reproductive cycle, large numbers of offspring, synchronization of oocyte maturation, and other life history characteristics. However, existing gene manipulation techniques are insufficient to accurately define gene functions. Although our previous investigations developed an RNA interference (RNAi) system in D. pulex, the possible time period of functional analysis was limited because the effectiveness of RNAi is transient. Thus, in this study, we developed a genome editing system for D. pulex by first microinjecting transcription activator-like effector nuclease (TALEN) mRNAs into early embryos and then evaluating TALEN activity and mutation phenotypes.
\end{abstract}

Results: We assembled a TALEN construct specific to the Distal-less gene (D/I), which is a homeobox transcription factor essential for distal limb development in invertebrates and vertebrates, and evaluated its activity in vitro by single-strand annealing assay. Then, we injected TALEN mRNAs into eggs within 1 hour post-ovulation. Injected embryos presented with defects in the second antenna and altered appendage development, and indel mutations were detected in D/l loci, indicating that this technique successfully knocked out the target gene.

Conclusions: We succeeded, for the first time in D. pulex, in targeted mutagenesis by use of Platinum TALENs. This genome editing technique makes it possible to conduct reverse genetic analysis in D. pulex, making this species an even more appropriate model organism for environmental, evolutionary, and developmental genomics.

Keywords: Daphnia pulex, Distal-less, Platinum TALEN, Gene disruption, Knock-out, Targeted mutagenesis, Gene manipulation, Genome editing

\section{Background}

Environmental cues constantly influence gene expression; hence interactions among them are indispensable for animal adaptations against changing environments. The water flea Daphnia pulex shows a striking ability to contend with environmental changes, resulting in various

\footnotetext{
* Correspondence: taisen@nibb.ac.jp

'Okazaki Institute for Integrative Bioscience, National Institute for Basic Biology, National Institutes of Natural Sciences, Higashiyama 5-1, Myodaiji, Okazaki, Aichi 444-8787, Japan

${ }^{2}$ Faculty of Life Science, Graduate University for Advanced Studies (SOKENDAI), 5-1 Higashiyama, Myodaiji, Okazaki, Aichi 444-8787, Japan Full list of author information is available at the end of the article
}

adaptive phenotypes involving traits such as body size, longevity, behavior, and morphology [1]. It is well known that daphnids occupy a key position as the intermediate link between primary productivity and top predators in the aquatic food chain, and they also serve as an environmental indicator organism because of their high sensitivity to water quality [2]. These characteristics, in addition to their short life cycle, large brood sizes, and synchronization of oocyte maturation, make them valuable for environmental, evolutionary, and developmental genomics studies. Furthermore, the D. pulex genome has been sequenced [3], which facilitates the identification of candidate genes involved in 
their unique biological attributes. The analysis of transcriptomics, proteomics, and metabolomics also showed profiles of a large number of putative factors involved in their unique life history characteristics (cf. $[4,5])$. The $D$. pulex genome possesses as many as about 31,000 genes; this large suite of genes may provide the arsenal responsible for the organism's responsiveness to environmental challenges [3]. So far, however, there are no effective methods for manipulating genes, and only transient analysis of gene function by the RNA interference (RNAi) method [6] is available. The RNAi system in D. pulex suffers from several weaknesses, including incomplete silencing, transient effects, and limited analyzable stages. Because of these situations, even in the post-genomic era, the establishment of a gene manipulation technique has been eagerly anticipated to address the characterization of gene function in D. pulex.

There are three main types of artificial sequence-specific nucleases based on the source mechanism of DNA binding that guides nuclease activity to a genomic target: zincfinger nucleases (ZFNs), transcription activator-like effector nucleases (TALENs), and clustered regularly interspaced short palindromic repeats (CRISPR) [7]. In this study, we chose TALEN to edit the $D$. pulex genome, although each method has both advantages and disadvantages in terms of cost, sequence-specificity, off-target effects, and so on $[8,9]$. The transcription activator-like effectors (TALEs) were first discovered in the plant pathogen Xanthomonas sp., and are composed of a conserved central domain for site-specific DNA binding [10,11]. The DNA-binding domain consists of varying numbers of tandem repeats of a 34-amino acid monomer, which specifies the DNA-binding sequence by its 12th and 13th amino acids, called repeat-variable diresidues (RVDs). RVDs specifically recognize a single nucleotide with the following codes: $\mathrm{NG}=\mathrm{T}, \mathrm{HD}=\mathrm{C}, \mathrm{NI}=\mathrm{A}$, $\mathrm{NN}=\mathrm{G}$ or A $[10,12,13]$. Sakuma et al. [14] demonstrated that TALENs with periodically-patterned repeat variants harboring non-RVD variations, called Platinum TALENs, showed higher activities than TALENs without non-RVD variations (so-called Golden TALENs). TALENs are artificially generated by fusing TAL effector DNA-binding domains to a Fok I nuclease domain, and successfully harnessed to custom-designed sequence-specific nucleases $[15,16]$. TALENs induce DNA double-strand breaks (DSBs) that can be repaired by the error-prone non-homologous end joining (NHEJ) system to cause insertion and/or deletion mutations at targeted genomic loci.

TALEN-mediated gene targeting has been applied in a great number of vertebrates and invertebrates $[7,9,17]$. In arthropods, it has been reported in insect species including flies [18], mosquitos [19,20], crickets [21], and silkworms [22]. Very recently, mutagenesis of an eye development gene was achieved in one congener Daphnia magna using the CRISPR/Cas system [23]. However, the conditions developed in $D$. magna are not directly applicable to $D$. pulex, possibly because of the difference in egg size and contents, and developmental duration, as described in our previous study [6].

Distal-less $(D l l)$ and its homologs, Dlx genes, which function as homeodomain transcription factors, play one of the major roles in distal limb development throughout the animal kingdom [24]. Reduction of Dll activity caused defects of distal appendage segments in arthropods, resulting in the production of an easily recognizable phenotype (cf. $[25,26])$. In addition, the results of $D l l$ RNAi in $D$. pulex [6] provide possible comparative data regarding phenotype, which is why this endogenous developmental gene was selected as a target for proof-of-principle TALEN in D. pulex.

The goal of this study was to develop a targeted gene disruption system by TALEN-mediated artificial DSBs in $D$. pulex. A TALEN target site was designed and assembled, and then TALEN mRNAs were microinjected into embryos to successfully induce insertion and/or deletion mutations.

\section{Results and discussion}

\section{Construction and evaluation of DII TALENs}

We first cloned and sequenced the partial sequence of the Dll gene in D. pulex, and then designed two pairs of TALENs, Dll_A and Dll_B, targeted to the first exon to induce a nonsense mutant protein (Figure 1). After constructing the TALENs by the Platinum Gate TALEN Kit, the activities of TALENs were evaluated by human cell-based single-strand annealing (SSA) assay [27] (Figure 2). Both Dll_A and Dll_B TALENs showed higher scores than the positive control ZFN [28], suggesting that both TALENs have DSB-inducing activities. We have evaluated the TALEN activities using human cells for various animal applications including sea urchin [29] and fruit fly [27,30], suggesting that the activity score measured in human cells is, in principle, a good indication of the level of inducing DSBs in a wide range of animals. SSA assay can directly be performed with the constructed TALEN plasmids in human cells, so that we can easily know the quality of the constructed TALENs before making mRNAs and injecting them into the animals. We thus think the validation process with human cellbased SSA assay is beneficial to apply TALENs in animals including D. pulex.

\section{Detection of mutations and their sequences}

Two pairs of Dll TALEN mRNAs were microinjected into eggs just after ovulation, and the genome modification efficiencies in vivo were evaluated by a T7 endonuclease I (T7EI) assay. We found that a signal intensity of undigested band in the TALEN-injected embryos was weaker than that in uninjected embryos (Figure 3A). Furthermore, multiple digested bands were detected in Dll_B-injected embryos, whereas only a slightly separated band was detected in 


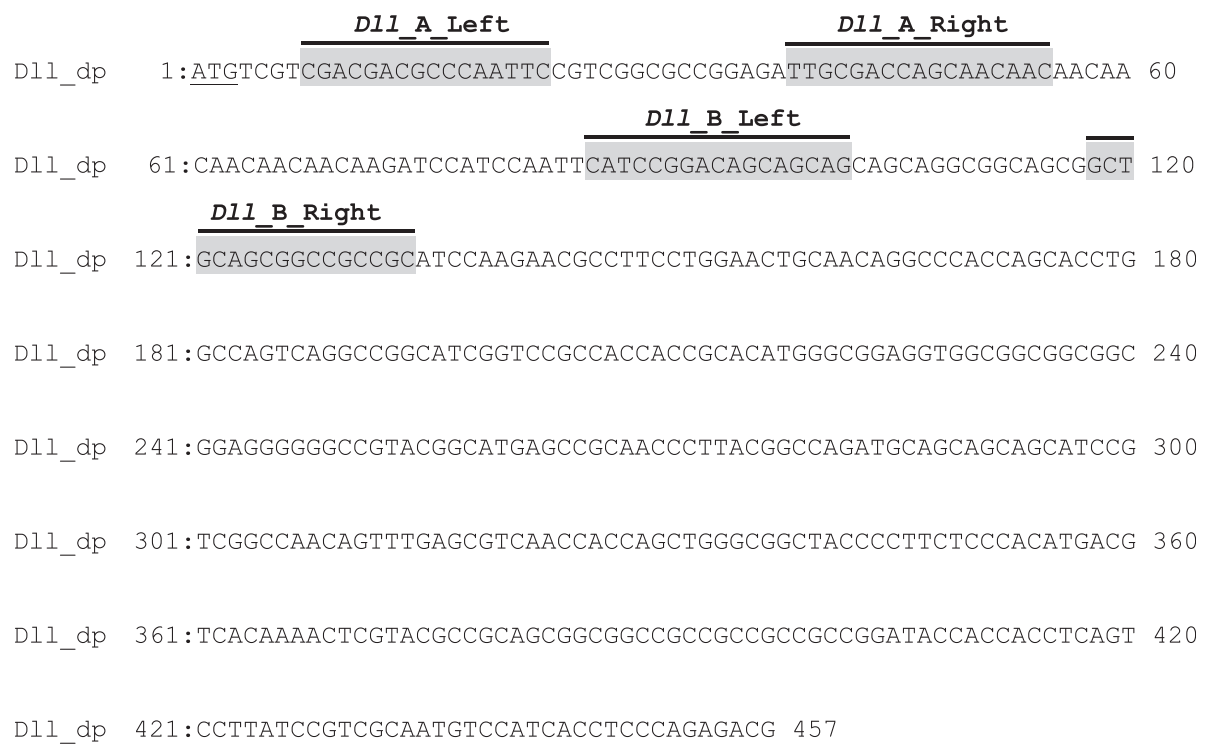

Figure 1 The first exon nucleotide sequence of the $D$. pulex DII gene. Two pairs of TALENs, DII_A and DII_B, were designed in the first exon (highlighted in gray). The spacer regions of DII_A and DII_B each contain 15 bp nucleotides. Underline indicates ATG start codon.

Dll_A-injected embryos. We thus concluded that Dll_B exhibits higher genome modification efficiencies rather than Dll_A.

To investigate the successful genome modifications of the $\mathrm{F}_{0}$ founder line, the genomic region surrounding the TALEN target was amplified and subcloned. Sequencing analysis revealed that TALENs predominantly induce small indel mutations ranging in size from 6-13 bp (Figure 3B),

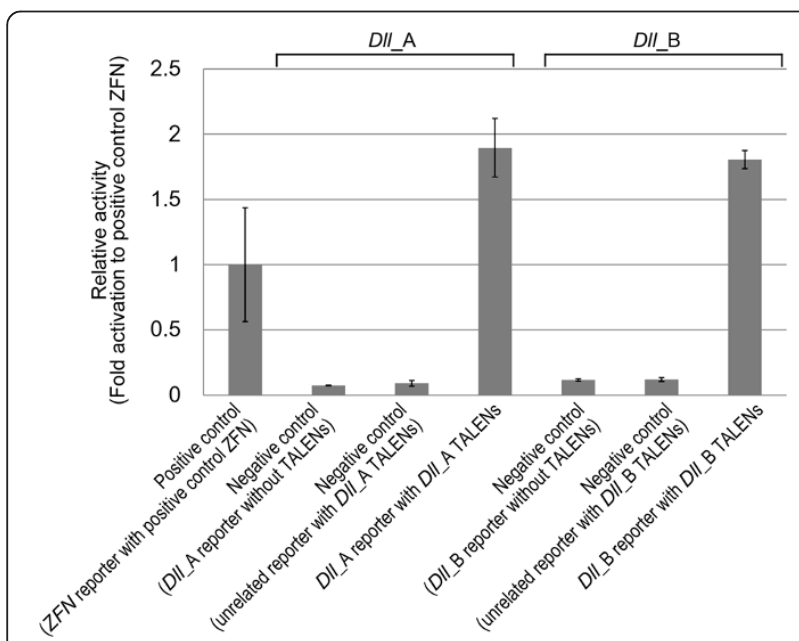

Figure 2 Activity evaluation of DII_A and DII_B TALENs by SSA assay. TALEN plasmids and the corresponding reporter plasmid, TALEN plasmids and unrelated reporter plasmid, or the reporter plasmid without TALEN plasmids were transfected into HEK293T cells. After the luciferase assay, fold activation scores against the positive control ZFN (pSTL-ZFA36 with the corresponding reporter) [28] were calculated. Data are expressed as means \pm SEM $(n=3)$. which is similar to results obtained in other arthropods [18-22]. In Dll_A-injected $F_{0}$ founders, only two types of mutations, 6- and 9-nucleotide deletions, were detected. These mutations did not cause a frame shift and growth defect (see next section), suggesting that the gene function was not compromised. On the other hand, seven types of mutations were detected in Dll_B-injected $\mathrm{F}_{0}$ founders, five of which induced a frame shift leading to the appearance of a stop codon in the first exon. These mutations are predicted to result in a very short protein (83 amino acids) without a homeodomain, which is a functional DNAbinding domain and highly conserved among other arthropods. The variations of mutation patterns in $D l l \_B$-injected $\mathrm{F}_{0}$ founders were higher than those in Dll_A-injected $\mathrm{F}_{0}$ founders, as expected from the T7EI assay. Based on these results, we conclude that the T7EI assay is a quick and easy method for estimating TALEN in vivo activity in $\mathrm{F}_{0}$ embryos of $D$. pulex.

To investigate whether these mutations were heritable, we then cloned and sequenced the target genomic region from $\mathrm{F}_{2}$ embryos. DNA sequencing revealed that inherited modifications existed in the two and one $F_{2}$ lines injected with Dll_A and B, respectively (Figure 3C). Progeny from the same $\mathrm{F}_{0}$ founder had the same mutation genotype, indicating that the $F_{0}$ founder carried a single germline mutation, not a mosaic germline mutation. Because cleavage proceeds without cytokinesis during injection period [31] and TALEN mRNAs can easily diffuse throughout the egg, it is considered that this method is able to induce mutation at very early developmental stages and easily establish mutant lines having a single germline 


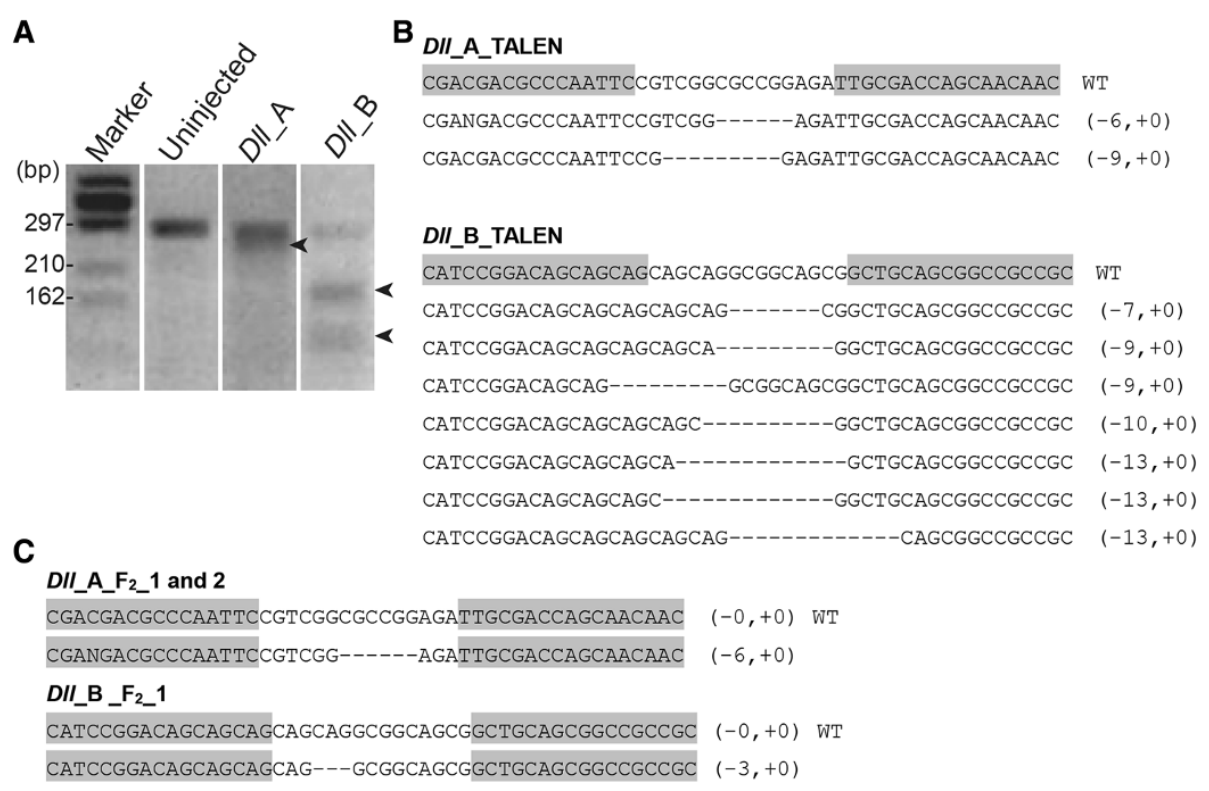

Figure 3 Detection of TALEN-induced mutations and their sequences. (A) Mutations in D/l were detected by T7El assay. Arrowheads indicate the cleavage products generated in T7El assay of $F_{0}$ founders. (B) Sequence analysis of D/l mutant alleles in $F_{0}$ founders induced by TALENs. The wild type (WT) sequence is shown at the top with the TALEN-binding sites (highlighted in gray). The number of nucleotides deleted (-) and inserted (+) is indicated in parentheses. (C) Sequence analysis of D/l mutant alleles in $F_{2}$ embryos. Binding sites and insertions/deletions indicated as in (B).

mutation, without a need for complicated screening. In other words, mutation analysis can be carried out in a mere generation.

\section{Phenotype of DII TALEN in D. pulex}

Results from TALEN experiments are summarized in Table 1. Compared to uninjected embryos, the low viability of the embryos injected with $D l l$ TALEN mRNAs may be partly attributed to damage due to microinjection and/ or toxicity of TALEN mRNAs, as well as the embryonic lethality of $D l l$ knock-out mutants. The difference of viability and/or morphological phenotype among embryos injected with $D l l \_$A or $D l l \_B$ might depend on their genetic phenotype, such as non-mosaic/mosaic, monoallelic/ biallelic, and non-frame shift/frame shift mutations. When a half concentration of $D l l \_B(250 \mathrm{ng} / \mu \mathrm{l})$ was injected, viability of injected embryos increased by $7.5 \%$ and two individuals did not have any prominent altered phenotypes, suggesting dose dependency of the TALENs on viability and phenotypes.

Morphologically, the Dll_A injected embryos did not show any prominent altered phenotypes, and both homozygous and heterozygous non-frame shift mutations were detected in $D l l$ loci. Hence, we assessed the phenotype of $D l l \_B$-injected $\mathrm{F}_{0}$ founders hereafter. Similar to the RNAi knockdown phenotype of $\mathrm{Dll}$ [6], Dll_B-injected $\mathrm{F}_{0}$ founders displayed various degrees of defects in second antennae, appendages, ocellus, abdominal claw, and abdominal setae, all tissues in which Dll is normally expressed (Figure 4 and Additional file 1: Figure S1). In the second antennae, the degree of the segment truncation was variably detected, ranging from severe (peduncle alone, lacking dorsal and ventral rami) to mild (peduncle and deficient rami). The first to fifth thoracic appendages, including each exopodite, were shortened. Moreover, a loss of the ocellus and abdominal setae, and minimized abdominal claw, were observed in Dll_B-injected embryos. We found that the mutants with severe defects in the second antenna and appendage development have biallelic mutations, failed to molt, and died before becoming adults. The lethality of $\mathrm{Dll}$ knock-out mutants was in accordance with previous studies on insects [25,32]. This easy and efficient induction of biallelic mutations by TALENs provides a valuable tool for parthenogenetic

Table 1 Summary of TALEN results

\begin{tabular}{llllll}
\hline Platinum TALENs & mRNAs concentration $(\mathbf{n g} / \boldsymbol{\mu l})$ & Injected embryos & Juveniles & Viability $(\%)$ & Shortened 2 $^{\text {nd }}$ antennae \\
\hline DII_A & 500 & 88 & 36 & 40.9 & 0 \\
DII_B & 500 & 206 & 63 & 30.6 & 63 \\
& 250 & 134 & 51 & 38.1 & 49 \\
Uninjected & - & 130 & 107 & 82.3 & 0 \\
\hline
\end{tabular}




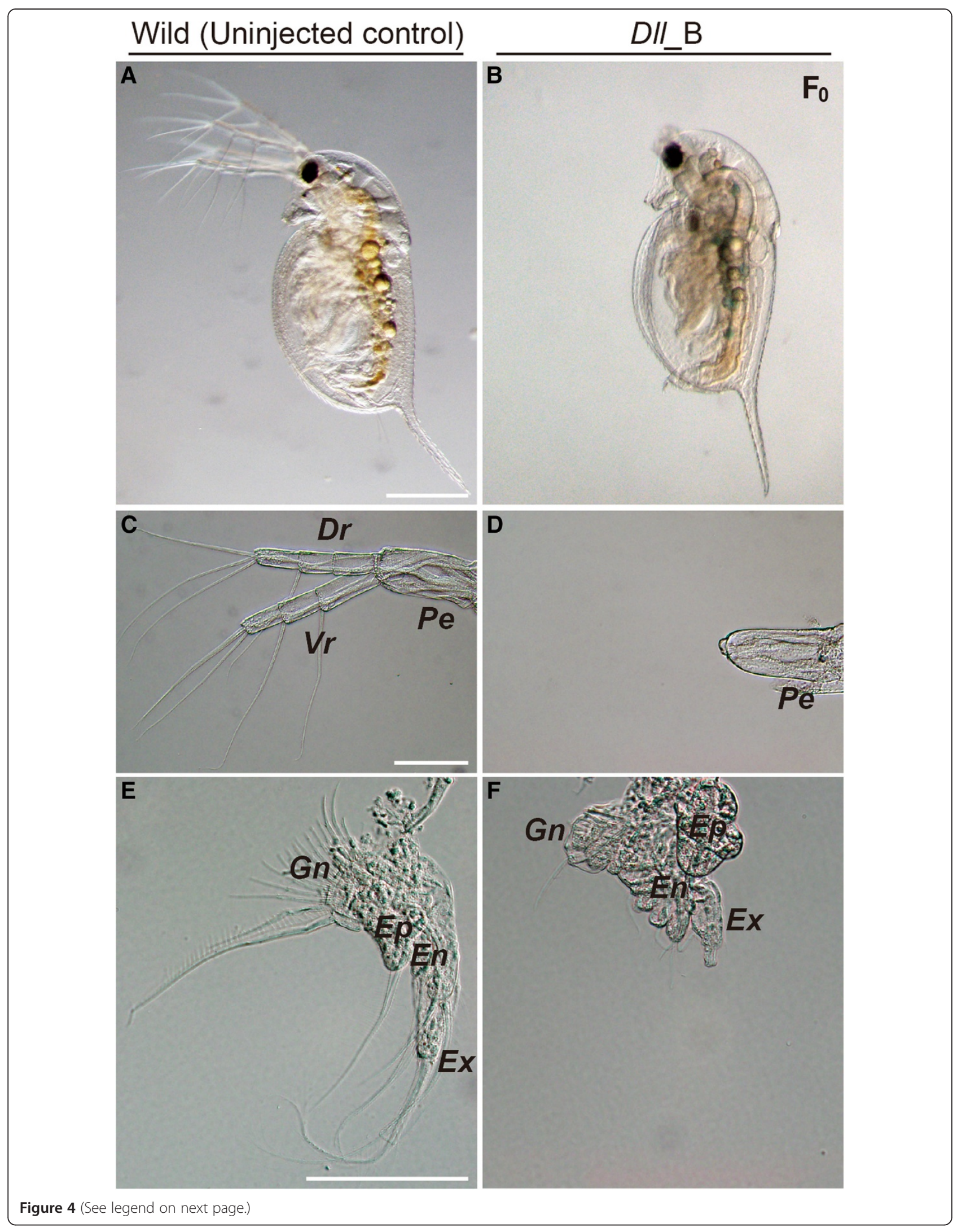


animals, including Daphnia, which are unable or poorly suited for conducting crossing experiments. In cases where monoallelic mutants are obtained, it is possible that the mutant line could be maintained and embryos reinjected with TALEN mRNAs to induce biallelic mutations.

Our results demonstrated that TALENs worked in $D$. pulex to induce heritable mutations into the endogenous genes. Moreover, TALENs can be used to induce more complicated mutagenesis other than micro-deletions or insertions. For example, Ma et al. [22] showed that simultaneous expression of two pairs of TALENs generates heritable large chromosomal deletions. Furthermore, Bedell et al. [33] and $\mathrm{Zu}$ et al. [34] demonstrated that co-injection of TALEN mRNAs and single-stranded oligodeoxyribonucleotides or donor vectors induced knock-in of small and large DNA fragments, respectively, into target loci via homologous recombination. All these technique based on TALENs will enable us to accurately define gene functions in $D$. pulex in future investigations.

\section{Conclusions}

We developed a TALEN-mediated gene targeting model in D. pulex. Targeted mutagenesis can be attained in early embryos by injecting the right and left TALEN mRNAs into eggs within 1 hour post-ovulation. The genome editing by Platinum TALENs presented here, together with the full-genome sequence and other developed molecular tools, may significantly promote the genetic tractability of D. pulex as an important model for environmental, evolutionary, and developmental genomics.

\section{Methods}

\section{Daphnia strain and culture conditions}

The D. pulex strain [West Trenton (WTN6), collected in May 2006 by Sarah Schaak] was obtained from the Center for Genomics and Bioinformatics (Indiana University, USA). The strains were maintained in dechlorinated tap water, which was aerated and filtered through activated carbon for 2 weeks, at $18^{\circ} \mathrm{C}$ under artificial light conditions of $14 \mathrm{~h}$ light and $10 \mathrm{~h}$ dark to maintain reproduction. A $0.01-\mathrm{ml}$ suspension of $10^{9}$ cells $/ \mathrm{ml}$ Chlorella vulgaris was added every day to each culture (20-25 individuals/L). For rearing embryos, M4 culture medium (M4) was prepared using MilliQ water [35].

\section{Cloning of Distal-less}

Total RNA was extracted using an ISOGEN kit (NIPPON GENE, Tokyo, Japan), and converted to cDNA using
Superscript III and random primers (Life Technologies, Carlsbad, CA, USA) according to the manufacturer's protocol. The Dll fragment was PCR amplified from the cDNA using a set of primers designed from the Dll sequence retrieved from wFleaBase http://wfleabase.org/ (Additional file 2: Table S1). Subsequently, the cDNA fragments were cloned into the pGEM-T Easy vector (Promega, Madison, WI, USA) according to the manufacturer's instructions. Plasmids were sequenced using Sanger techniques that included the Big Dye terminator Ver. 3.1 (Life Technologies) on an ABI 3100 Avant or ABI 3130 Genetic Analyser DNA sequencer (Applied Biosystems Japan Ltd, Tokyo, Japan).

\section{TALEN target site design and assembly}

The first exon sequences of $D l l$ gene were scanned for potential TALEN target sites, which were identified using the TALEN Targeter program at https://tale-nt.cac.cornell. edu/node/add/talen. The following parameters were used: 1) spacer length: $12-16 ; 2$ ) repeat array length of $15-20 ; 3$ ) G substitute: NN; 4) Filter Options: Show all TALEN pairs (include redundant TALENs); 5) Streubel et al. guidelines: On. The Platinum Gate TALEN construction system described in Sakuma et al. [14] was used to assemble two pairs of TALENs, $D l l_{-}$A and $D l l_{-}$B (Figure 1), using the Platinum Gate TALEN Kit (Addgene, cat\#1000000043). Assembled RVD repeats were as follows: HD NN NI HD NN NI HD NN HD HD HD NI NI NG NG HD for the left $D l l \_$A TALEN, NN NG NG NN NG NG NN HD NG NN NN NG HD NN HD NI NI for the right Dll_A TALEN, HD NI NG HD HD NN NN NI HD NI NN HD NI NN HD NI NN for the left Dll_B TALEN, and NN HD NN NN HD NN NN HD HD NN HD NG NN HD NI NN HD for the right Dll_B TALEN. ptCMV-153/47-VR vectors (Addgene, included in the Platinum Gate TALEN Kit) were used as destination vectors.

\section{Single-strand annealing (SSA) assay in human cells}

Construction of the reporter plasmids and the SSA assay was performed as previously reported [27]. Briefly, the oligonucleotides listed in Additional file 3: Table S2 were annealed and inserted into pGL4-SSA vector (Addgene, Plasmid 42962) to construct the reporter plasmids. The TALEN plasmids and/or reporter plasmids were transfected into HEK293T cells using Lipofectamine LTX (Life Technologies) according to the manufacturer's instruction. At 24 hours post-transfection, a luciferase assay was performed using Dual-Glo Luciferase Assay System (Promega) and intensity of luminescence was 
measured with TriStar LB 941 plate reader (Berthold Technologies, Bad Wildbad, Germany).

\section{Injection of TALEN mRNAs into embryos}

TALEN plasmid templates were linearized with Sma I (TaKaRa Bio, Shiga, Japan) and then transcribed in vitro using the mMESSAGE mMACHINE T7 Ultra kit (Ambion, Austin, TX, USA) according to the manufacturer's protocol. The resultant mRNAs were purified using a NucAway Spin Columns (Ambion) and extracted with water-saturated phenol/chloroform, and finally resuspended in RNase-free water. Equal amounts of the right and left TALEN mRNAs were mixed to a final concentration of 500 and $250 \mathrm{ng} / \mu \mathrm{l}$ for microinjection and stored at $-80^{\circ} \mathrm{C}$ until use. TALEN mRNAs were injected into the embryos as described previously [6].

\section{T7 endonuclease I (T7EI) assay}

T7EI is a mismatch-resolving enzyme that can recognize heteroduplex DNAs and cleave DNA at single base pair mismatches. Thus TALEN-induced indel mutations can be detected. A pool of thirteen embryos collected at $48 \mathrm{~h}$ following injection with $D l l_{\text {_ A }}$ or $D l l_{-} \mathrm{B}$ was used. Genomic DNA was isolated from TALEN-injected and uninjected embryos using DNeasy Blood and Tissue kit (Qiagen, Hilden, Germany). Additional file 2: Table S1 shows primers used to amplify the region containing the $D l l$ target site from genomic DNA for T7EI assay and mutation analysis. PCR was performed using TaKaRa Ex Taq (TaKaRa Bio). Amplification conditions were: $98^{\circ} \mathrm{C}$ for $10 \mathrm{~s}, 56^{\circ} \mathrm{C}$ for $30 \mathrm{~s}, 72^{\circ} \mathrm{C}$ for 20 s, 45 cycles. T7 endonuclease I (New England Biolabs, Beverly, MA, USA) was added to PCR fragments and incubated at $37^{\circ} \mathrm{C}$ for $60 \mathrm{~min}$. The samples were electrophoresed using $2 \%$ agarose gels.

\section{Mutation analysis}

To confirm the presence of TALEN-mediated mutations, genomic DNA of each individual was isolated separately from twelve $F_{0}$ embryos and nine $F_{2}$ embryos injected with $D l l \_$A or $D l l \_$B as described above. The target genomic region was amplified with TaKaRa Ex Taq (TaKaRa Bio). Amplification conditions were: $98^{\circ} \mathrm{C}$ for $1 \mathrm{~min} ; 35$ cycles of $98^{\circ} \mathrm{C}$ for $10 \mathrm{~s}, 56^{\circ} \mathrm{C}$ for $30 \mathrm{~s}, 72^{\circ} \mathrm{C}$ for $20 \mathrm{~s} ; 72^{\circ} \mathrm{C}$ for $1 \mathrm{~min}$. The resulting fragments were subcloned into the pGEM-T Easy vector (Promega) and then sequenced as described above. For observation of morphological changes, first instar animals were examined under a stereomicroscope (M165 FC; Leica Microsystems $\mathrm{GmbH}$, Wetzlar, Germany), and then fixed in ethanol and dissected with a pair of needles. The specimens were mounted in glycerin, observed by Nomarski differential interference contrast microscopy (Axioplan 2; Zeiss, Oberkochen, Germany), and recorded by digital camera (DP72; Olympus). The terminology used herein for morphology follows the usage of Stachowitsch [36].

\section{Additional files}

Additional file 1: Figure S1. Phenotypes of DII_B mRNAs injected juveniles. The left and right columns show representative phenotypes of uninjected controls and individuals injected with DII_B mRNAs, respectively. (A, B) First thoracic limb (T1). The exopodite and endopodite were shortened by DII_B TALEN mRNAs. (C, D) Third and fourth thoracic limbs (T3/4), having the same morphology. The exopodite was shrunk in DII_B-injected juveniles. (E, F) Fifth thoracic limb (T5). The exopodite was shortened by DII_B mRNAs. (G, H) Lateral view of the rostrum and head; an arrowhead indicates an ocellus. (I, J) Lateral view of abdomen; an arrow and arrowhead show an abdominal claw and abdominal seta, respectively. Ep, epipodite; $E n$, endopodite; $E x$, exopodite; $F c$, filter comb. Scale bars $=100 \mu \mathrm{m}$.

Additional file 2: Table S1. The list of primer sequences.

Additional file 3: Table S2. Oligonucleotides for construction of reporter plasmids.

\section{Competing interests}

The authors declare that they have no competing interests.

\section{Authors' contributions}

$\mathrm{CH}$ and $\mathrm{YO}$ designed the work, performed and analyzed the experiments; $\mathrm{CH}, \mathrm{TS}, \mathrm{TY}$ and TI wrote the paper. TS and TY performed TALEN construction and SSA assay. KT and SM discussed and commented on results. All authors have read and approved the final manuscript.

\section{Acknowledgments}

Daphnia pulex genomic sequence data were produced by The Center for Genomics and Bioinformatics at Indiana University and distributed via wFleaBase in collaboration with the Daphnia Genomics Consortium https:// wiki.cgb.indiana.edu/display/DGC/Home. We thank Brenna Doheny for reviewing and editing the manuscript; members of the Iguchi laboratory for helpful advice and discussions; members of Yamamoto's laboratory for assistance in TALEN construction. This work was supported by a JSPS Research Fellowship for Young Scientists to CH (No.12J04065), KT (No.12J05579), JSPS KAKENHI Grant Number 26840115 to $\mathrm{CH}$, a grant from Ministry of the Environment, Japan and MEXT KAKENHI Grant Numbers 24657022, 24370029 to TI. Our work benefits from, and contributes to, the Daphnia Genomics Consortium.

\section{Author details}

${ }^{1}$ Okazaki Institute for Integrative Bioscience, National Institute for Basic Biology, National Institutes of Natural Sciences, Higashiyama 5-1, Myodaiji, Okazaki, Aichi 444-8787, Japan. ${ }^{2}$ Faculty of Life Science, Graduate University for Advanced Studies (SOKENDAI), 5-1 Higashiyama, Myodaiji, Okazaki, Aichi 444-8787, Japan. ${ }^{3}$ Department of Mathematical and Life Sciences, Graduate School of Science, Hiroshima University, Higashi-Hiroshima 739-8526, Japan.

Received: 26 July 2014 Accepted: 28 October 2014

Published online: 18 November 2014

\section{References}

1. Tollrian R, Harvell CD: The Ecology And Evolution Of Inducible Defences. Princeton: Princeton University Press; 1999.

2. Dodson SI, Hanazato T: Commentary on effects of anthropogenic and natural organic chemicals on development, swimming behavior, and reproduction of Daphnia, a key member of aquatic ecosystems. Environ Health Perspect 1995, 103:7-11.

3. Colbourne JK, Pfrender ME, Gilbert D, Thomas WK, Tucker A, Oakley TH, Tokishita S, Aerts A, Arnold GJ, Basu MK, Bauer DJ, Cáceres CE, Carmel L, Casola C, Choi J-H, Detter JC, Dong Q, Dusheyko S, Eads BD, Fröhlich T, GeilerSamerotte KA, Gerlach D, Hatcher P, Jogdeo S, Krijgsveld J, Kriventseva EV, Kültz D, Laforsch C, Lindquist E, Lopez J, et al: The ecoresponsive genome of Daphnia pulex. Science 2011, 331:555-561. 
4. Gard AL, Lenz PH, Shaw JR, Christie AE: Identification of putative peptide paracrines/hormones in the water flea Daphnia pulex (Crustacea; Branchiopoda; Cladocera) using transcriptomics and immunohistochemistry. Gen Comp Endocr 2009, 160:271-287.

5. Dircksen H, Neupert S, Predel R, Verleyen P, Huybrechts J, Strauss J, Hauser F, Stafflinger E, Schneider M, Pauwels K, Schoofs L, Grimmelikhuijzen CJP: Genomics, transcriptomics, and peptidomics of Daphnia pulex neuropeptides and protein hormones. J Proteome Res 2011, 10:4478-4504.

6. Hiruta C, Toyota K, Miyakawa H, Ogino Y, Miyagawa S, Tatarazako N, Shaw $J R$, Iguchi T: Development of a microinjection system for RNA interference in the water flea Daphnia pulex. BMC Biotechnol 2013, 13:96

7. Sakuma T, Woltjen K: Nuclease-mediated genome editing: At the front-line of functional genomics technology. Develop Growth Differ 2014, 56:2-13.

8. DeFrancesco L: Move over ZFNs. Nat Biotechnol 2011, 29:681-684.

9. Gaj T, Gersbach CA, Barbas CF: ZFN, TALEN, and CRISPR/Cas-based methods for genome engineering. Trends Biotechnol 2013, 31:397-405.

10. Boch J, Scholze H, Schornack S, Landgraf A, Hahn S, Kay S, Lahaye T, Nickstadt A, Bonas U: Breaking the code of DNA binding specificity of TAL-Type III effectors. Science 2009, 326:1509-1512.

11. Boch J, Bonas U: Xanthomonas AvrBs3 family-type III effectors: discovery and function. Annu Rev Phytopathol 2010, 48:419-436.

12. Moscou MJ, Bogdanove AJ: A simple cipher governs DNA recognition by TAL effectors. Science 2009, 326:1501.

13. Bogdanove AJ, Voytas DF: TAL effectors: Customizable proteins for DNA targeting. Science 2011, 333:1843-1846.

14. Sakuma T, Ochiai H, Kaneko T, Mashimo T, Tokumasu D, Sakane Y, Suzuki K, Miyamoto T, Sakamoto N, Matsuura S, Yamamoto T: Repeating pattern of non-RVD variations in DNA-binding modules enhances TALEN activity. Sci Rep 2013, 3:3379.

15. Miller JC, Tan S, Qiao G, Barlow KA, Wang J, Xia DF, Meng X, Paschon DE, Leung E, Hinkley SJ, Dulay GP, Hua KL, Ankoudinova I, Cost GJ, Urnov FD, Zhang HS, Holmes MC, Zhang L, Gregory PD, Rebar EJ: A TALE nuclease architecture for efficient genome editing. Nat Biotechnol 2011, 29:143-148.

16. Cermak T, Doyle EL, Christian M, Wang L, Zhang Y, Schmidt C, Baller JA, Somia NV, Bogdanove AJ, Voytas DF: Efficient design and assembly of custom TALEN and other TAL effector-based constructs for DNA targeting. Nucleic Acids Res 2011, 39:e82.

17. Joung JK, Sander JD: TALENs: a widely applicable technology for targeted genome editing. Nat Rev Mol Cell Biol 2013, 14:49-55.

18. Liu J, Li C, Yu Z, Huang P, Wu H, Wei C, Zhu N, Shen Y, Chen Y, Zhang B, Dend WM, Jiao R: Efficient and specific modifications of the Drosophila genome by means of an easy TALEN strategy. J Genet Genomics 2012, 39:209-215.

19. Aryan A, Anderson MA, Myles KM, Adelman ZN: TALEN-based gene disruption in the dengue vector Aedes aegypti. PLoS One 2013, 8:e60082.

20. Smidler AL, Terenzi O, Soichot J, Levashina EA, Marois E: Targeted mutagenesis in the malaria mosquito using TALE nucleases. PLOS One 2013, 8:e74511.

21. Watanabe T, Ochiai H, Sakuma T, Horch HW, Hamaguchi N, Nakamura T, Bando T, Ohuchi H, Yamamoto T, Noji S, Mito T: Non-transgenic genome modifications in a hemimetabolous insect using zinc-finger and TAL effector nucleases. Nat Commun 2012, 3:1017.

22. Ma S, Zhang S, Wang F, Liu Y, Liu Y, Xu H, Liu C, Lin Y, Zhao P, Xia Q: Highly efficient and specific genome editing in silkworm using custom TALENs. PLoS One 2012, 7:e45035.

23. Nakanishi T, Kato Y, Matsuura T, Watanabe H: CRISPR/Cas-mediated targeted mutagenesis in Daphnia magna. PLoS One 2014, 5:e98363.

24. Panganiban G, Rubenstein JLR: Developmental functions of the Distal-less/ Dlx homeobox genes. Development 2002, 129:4371-4386.

25. Beermann A, Jay DG, Beeman RW, Hulskamp M, Tautz D, Jurgens G: The Short antennae gene of Tribolium is required for limb development and encodes the orthologue of the Drosophila Distal-less protein. Development 2001, 128:287-297.

26. Liubicich DM, Serano JM, Pavlopoulos A, Kontarakis Z, Protas ME, Kwan E, Chatterjee S, Tran KD, Averof M, Patel NH: Knockdown of Parhyale Ultrabithorax recapitulates evolutionary changes in crustacean appendage morphology. Proc Natl Acad Sci U S A 2009, 106:13892-13896.

27. Sakuma T, Hosoi S, Woltjen K, Suzuki K, Kashiwagi K, Wada H, Ochiai H, Miyamoto T, Kawai N, Sasakura Y, Matsuura S, Okada Y, Kawahara A, Hayashi S, Yamamoto T: Efficient TALEN construction and evaluation methods for human cell and animal applications. Genes Cells 2013, 18:315-326.
28. Ochiai H, Fujita K, Suzuki K, Nishikawa M, Shibata T, Sakamoto N, Yamamoto $\mathrm{T}$ : Targeted mutagenesis in the sea urchin embryo using zinc-finger nucleases. Genes Cells 2010, 15:875-885.

29. Hosoi S, Sakuma T, Sakamoto N, Yamamoto T: Targeted mutagenesis in sea urchin embryos using TALENs. Dev Growth Differ 2014, 56:92-97.

30. Kondo T, Sakuma T, Wada H, Akimoto-Kato A, Yamamoto T, Hayashi S: TALEN-induced gene knock out in Drosophila. Dev Growth Differ 2014, 56:86-91.

31. Hiruta C, Nishida C, Tochinai S: Abortive meiosis in the oogenesis of parthenogenetic Daphnia pulex. Chromosome Res 2010, 18:833-840.

32. Cohen SM, Jurgens G: Proximal-distal pattern formation in Drosophila: cell autonomous requirement for Distal-less gene activity in limb development. EMBO J 1989, 8:2045-2055.

33. Bedell V, Wang Y, Campbell JM, Poshusta TL, Starker CG, Krug RG II, Tan W, Penheiter SG, Ma AC, Leung AYH, Fahrenkrug SC, Carlson DF, Voytas DF, Clark KJ, Essner JJ, Ekker SC: In vivo genome editing using a highefficiency TALEN system. Nature 2012, 491:114-118.

34. Zu Y, Tong X, Wang Z, Liu D, Pan R, Li Z, Hu Y, Luo Z, Huang P, Wu Q, Zhu Z, Zhang B, Lin S: TALEN-mediated precise genome modification by homologous recombination in zebrafish. Nat Methods 2013, 10:329-331.

35. Elendt BP, Bias WR: Trace nutrient deficiency in Daphnia magna cultured in standard medium for toxity testing: effects of the optimization of culture conditions on life history parameters of $D$. magna. Water Res 1990, 24:1157-1167.

36. Stachowitsch M: The Invertebrates: An Illustrated Glossany. New York: Wiley-Liss Division; 1992

doi:10.1186/s12896-014-0095-7

Cite this article as: Hiruta et al:: Targeted gene disruption by use of transcription activator-like effector nuclease (TALEN) in the water flea Daphnia pulex. BMC Biotechnology 2014 14:95.

\section{Submit your next manuscript to BioMed Central and take full advantage of:}

- Convenient online submission

- Thorough peer review

- No space constraints or color figure charges

- Immediate publication on acceptance

- Inclusion in PubMed, CAS, Scopus and Google Scholar

- Research which is freely available for redistribution

Submit your manuscript at www.biomedcentral.com/submit
C) Biomed Central 